ORIGINAL ARTICLE

\title{
Gastrin releasing peptide and gastrin releasing peptide receptor expression in gastrointestinal carcinoid tumours
}

\author{
N Scott, E Millward, E J Cartwright, S R Preston, P L Coletta
}

J Clin Pathol 2004;57:189-192. doi: 10.1136/jcp.2003.10660

See end of article for authors' affiliations

Correspondence to:

Correspondence to:
Dr N Scott, Department of Pathology, St James's

University Hospital, Leeds LS9 TTF, UK:

nigelscott50@hotmail.com

Accepted for publication 4 August 2003

\begin{abstract}
Aims: To establish whether gastrin releasing peptide (GRP) and the GRP receptor (GRPR) are expressed together in gastrointestinal carcinoid tumours.

Methods: Twenty six carcinoid tumours from the stomach, small intestine, appendix, and colorectum were investigated by immunohistochemistry for GRP and GRPR.

Results: GRP was detected in nine of 19 tumours and GRPR in 22 of 26 . Coexpression of both the ligand and receptor was seen in six of 19 cases. GRPR but not GRP was more strongly expressed in appendix and colonic tumours.

Conclusions: GRP and GRPR are produced by a large number of gastrointestinal carcinoid tumours. An autocrine/paracrine pathway may exist for GRP stimulated cell proliferation in some of these neoplasms, analogous to that seen in small cell anaplastic carcinoma of the lung.
\end{abstract}

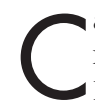

arcinoid tumours are low grade, malignant, epithelial neoplasms showing neuroendocrine differentiation. Recently, it has been suggested that the term carcinoid should be replaced by grade 1 (well differentiated) neuroendocrinecarcinoma. ${ }^{1}$ Carcinoid tumours most commonly arise in the bronchial tree and gastrointestinal tract $(25.1 \%$ and $73.7 \%$, respectively). In a recent large series of 5468 carcinoids at all sites, 3.2\% were located in the stomach, $2 \%$ in the duodenum, $26.2 \%$ in the small bowel, $18.9 \%$ in the appendix, and $21.3 \%$ in the colorectum. ${ }^{2}$ Their clinical behaviour is unpredictable and is influenced by site, tumour size, stage, evidence of metastasis, and histological features. ${ }^{3}$ Tumours located in the appendix are often found incidentally, are usually small, and seldom metastasise. ${ }^{4}$ In contrast, carcinoid tumours of the ileum and jejunum are frequently metastatic to regional lymph nodes and liver at the time of diagnosis. ${ }^{5}$ Gastric carcinoids may be either sporadic, solitary, and antral in location, or may develop in the context of hypergastrinaemia (for example, in the setting of pernicious anaemia), when they are often multiple, located in the corpus, and have a better prognosis. ${ }^{6}$ Most gastrointestinal carcinoid tumours are thought to develop from neoplastic transformation of endoderm derived neuroendocrine cells located in the gland or crypt epithelium. An exception to this is the appendix, where there is a population of subepithelial neuroendocrine cells closely related to lamina propria nerve fibres, and it is these that are believed to give rise to most appendix carcinoid tumours. ${ }^{7}$

\section{"Gastrin releasing peptide is a 27 amino acid peptide with a range of biological actions, including stimulation of gastrin release from antral $G$ cells, modulation of gastrointestinal motility, and stimulation of pancreatic exocrine secretion"}

This diversity in histogenesis, pathogenesis, and clinical behaviour might be expected to be reflected in the molecular biology of carcinoid tumours. Recent studies have demonstrated chromosome 11q deletions at the MENl gene locus in $48 \%$ of type 1 (hypergastrinaemia associated) gastric carcinoid tumours and in $33 \%$ of midgut carcinoids. ${ }^{8}$ Loss of heterozygosity at chromosome $18 \mathrm{q}$ is described in $67 \%$ of midgut tumours. ${ }^{9}$ A study of $\mathrm{X}$ chromosome deletion identified aberrations in $60 \%$ and $15 \%$ of malignant pancreatic and midgut endocrine tumours, respectively, compared with rates of $4.5 \%$ and $0 \%$ in clinically benign lesions. ${ }^{10}$ Immunohistochemical studies of tumour suppressor gene inactivation and oncogene expression have described overexpression of the anti-apoptotic bcl-2 protein in gastric endocrine cell hyperplasia, and loss of expression of E-cadherin in two thirds of malignant rectal carcinoid tumours. ${ }^{11}{ }^{12}$ Although p53 protein overexpression is unusual in most classic small intestinal tumours, it has been reported in up to $16 \%$ of colorectal carcinoids. ${ }^{13}{ }^{14}$ p5 53 mutations have also been found in $25 \%$ of goblet cell carcinoids of the appendix, in addition to a large number of typical appendix carcinoid tumours. ${ }^{15}$ In the same study, no k-ras mutations were identified in appendix lesions. Transforming growth factor $\alpha$ production has been detected in $72 \%$ of gastrointestinal carcinoid tumours. ${ }^{16}$ Most of these tumours also expressed its receptor, epidermal growth factor receptor. In addition to growth factors, carcinoid tumours express a wide range of peptide hormones, some of which have mitogenic effects. These include kinins, substance $\mathrm{P}$, gastrin, somatostatin, glucagon, corticotrophin, and calcitonin. An immunohistochemical study in 1984 by Bostwick et al demonstrated the production of gastrin releasing peptide (GRP), a mammalian homologue of bombesin, in seven of 10 small intestinal carcinoid tumours and four of five appendix tumours. ${ }^{17}$ GRP is a 27 amino acid peptide with a range of biological actions, including stimulation of gastrin release from antral G cells, modulation of gastrointestinal motility, and stimulation of pancreatic exocrine secretion. ${ }^{18}$ In addition to this it has a mitogenic, growth promoting effect on both normal tissues and tumour cell lines. In a rat model, GRP/bombesin stimulates the growth of normal pancreas, colon, and small intestine. ${ }^{19} 20$ In vitro it increases cell proliferation in human bronchial epithelial cells, breast cancer cells, and gastric and colon cancer cell lines. ${ }^{21-24}$ It appears to be an important autocrine/paracrine growth factor

Abbreviations: GRP, gastrin releasing peptide; GRPR, gastrin releasing peptide receptor 
for neuroendocrine tumours, including neuroblastoma and small cell anaplastic carcinoma of the lung. ${ }^{25} 26$

In our study, we aimed to confirm the expression of GRP in a series of gastrointestinal carcinoid tumours and use a novel polyclonal antibody to its receptor, GRPR, to establish whether an autocrine growth loop could also exist in gastrointestinal carcinoid tumours. To our knowledge, the status of the GRPR has not been examined previously in carcinoid tumours of the gastrointestinal tract, although aberrant expression of both GRP and GRPR has been described in over $80 \%$ of colon carcinomas. ${ }^{27}$

\section{MATERIALS AND METHODS}

Twenty six carcinoid tumours from 26 patients were identified from the pathology archive. Twelve were located in the small intestine, 10 in the appendix, three in the colon, and one in the stomach. A representative paraffin wax block containing tumour was selected for each case. In 11 cases, more than one paraffin wax block was investigated (total of 42 paraffin wax blocks). Immunohistochemistry was performed on paraffin wax sections using a biotinylated second antibody (swine antirabbit IgG; Dako, Ely, Cambridgeshire, UK) at a 1/200 dilution for 30 minutes at room temperature. The primary antibody used for detecting GRPR was a novel polyclonal rabbit antiserum raised against a synthetic peptide (residues 243-260), corresponding to a region of the third intracellular loop of human GRPR. This was used at a 1/100 dilution for one hour at room temperature. To detect GRP, a commercially available anti-GRP polyclonal antibody was used (Dako rabbit antihuman polyclonal; A0429). Before incubation with the primary antibody, endogenous peroxidase was blocked by immersion in hydrogen peroxide/ methanol for 10 minutes; antigen retrieval was performed by pressure cooking for one minute in citrate buffer $(\mathrm{pH} \mathrm{6)}$, and non-specific binding sites were blocked by incubation for 30 minutes in a $1 / 15$ dilution of normal swine serum/ phosphate buffered saline. To detect bound antibody, the sections were incubated for 30 minutes with streptavidinbiotin complex-horseradish peroxidase (strept ABC complexHRP; Dako). Diaminobenzidine was used as chromogen. Omission of the primary antibody was used as a negative control.

Immunohistochemical staining was scored semiquantitatively according to intensity and distribution in the following way: 1 , no staining; 2 , weak staining; 3 , strong staining; 1 , no cells stained; $2,<10 \%$ cells stained; $3,10-50 \%$ cells stained; $4,50-90 \%$ cells stained; and 5, all cells stained. Staining was interpreted by one investigator (NS). For the purposes of data presentation, tumours were considered positive if the sum score of intensity and distribution was $\geqslant 6$-that is, strong staining in at least $10 \%$ of cells or weak staining in over half of the tumour cell population. ${ }^{28}$ All 26 tumours were stained for GRPR. Nineteen of these were also examined for GRP. Insufficient tissue remained for GRP staining in seven cases. In the 11 tumours where more than one block of tissue was stained, the highest score was recorded.

The Wilcoxon rank sum test for non-paired samples was used to compare scores between tumours at different sites.

\section{RESULTS}

Staining for both GRP and GRPR was cytoplasmic (figs 1 and $2)$. No membrane staining was seen. In total, nine of 19 carcinoids expressed GRP, whereas 22 of 26 tumours expressed GRPR. Staining for GRP was often patchy and focal, whereas GRPR staining was usually more diffuse (figs 1 and 2). GRP staining was also seen in the nerve endings of the myenteric plexus. GRP and GRPR were not detected in normal endocrine cells of the small bowel or colon. Table 1 describes the results of immunostaining for individual

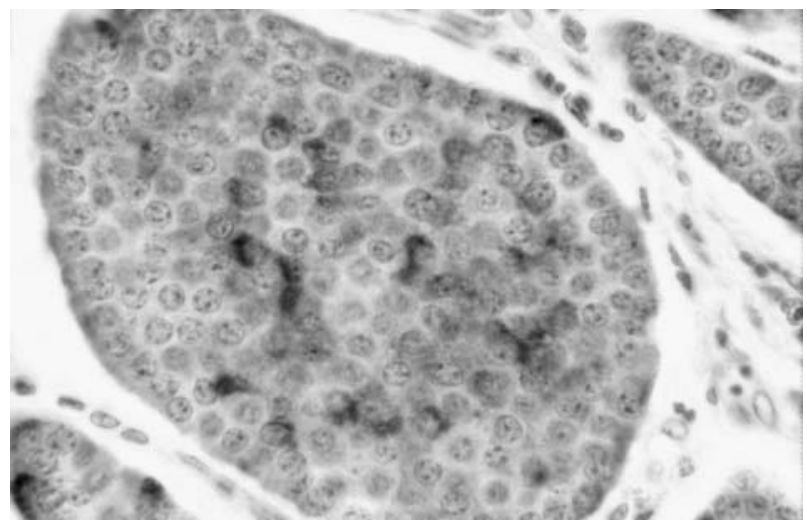

Figure 1 Gastrin releasing peptide expression in a small bowel carcinoid. Staining is heterogeneous and cytoplasmic (original magnification, $\times 100$ ).

tumour sites. In our study, GRP was less intensely expressed in appendix and colonic tumours than in small bowel neoplasms (mean scores, 2.86 and $1.00 v 5.53$ ). This did not reach significance however. GRPR was more strongly expressed in appendix and colonic tumours than in small bowel carcinoids (mean scores, 11 and $7.67 v 5.79$ ) and this was significant $(\mathrm{p}<0.05)$. Using our cutoff value of 6 , nine of the 12 small bowel tumours were positive compared with 12 of 13 colonic and appendix carcinoids. Furthermore, if only strength of staining was considered, 0 of 12 small bowel carcinoids showed strong $(3+)$ reactivity compared with eight of 13 appendix/colonic tumours. The single gastric carcinoid had a score of 6 for GRPR and 1 for GRP. Where more than one block from a tumour was immunostained, the immunoscore was concordant in 65\% of cases for GRPR and $79 \%$ of cases for GRP. In 19 cases where immunostaining was performed with both antibodies, only seven carcinoids were positive for both GRP and its receptor. This was mainly the result of absent or weak GRP expression in GRPR positive tumours.

Because the gene encoding GRPR is located on the $\mathrm{X}$ chromosome, we also tested whether receptor status was different between male and female patients; however, no significant difference was found (mean scores 6 and 5.9, respectively).

\section{DISCUSSION}

Our study shows for the first time that GRP, the mammalian homologue of bombesin, and its high affinity receptor GRPR, are expressed simultaneously in a proportion of gastrointestinal carcinoid tumours. GRP is a 27 amino acid peptide, encoded on chromosome 18, and synthesised as a larger precursor protein (prepro-GRP) with receptor binding activity localised to the $\mathrm{C}$-terminal end. ${ }^{29}$ The antibody that we used in our study recognises the mature amidated peptide, rather that its precursor. GRPR is a 384 amino acid transmembrane protein, encoded on the $\mathrm{X}$ chromosome, which belongs to a family of heptahelical G protein coupled receptors. ${ }^{30}$ For our study, we used a new rabbit polyclonal antibody raised against a synthetic peptide corresponding to a region of the third intracellular loop of human and mouse GRPR. Western blotting has shown that this antibody recognises a single $95 \mathrm{kDa}$ protein in mouse and human cell lines, corresponding to the expected size of fully glycosylated GRPR. Preincubation of this antibody with synthetic peptide has been shown to abolish immunocytochemical staining. GRP has several biological actions, including the stimulation of gastrointestinal hormone production, stimulation of pancreatic enzyme secretion, and regulation of intestinal motility. ${ }^{18}$ In addition, 


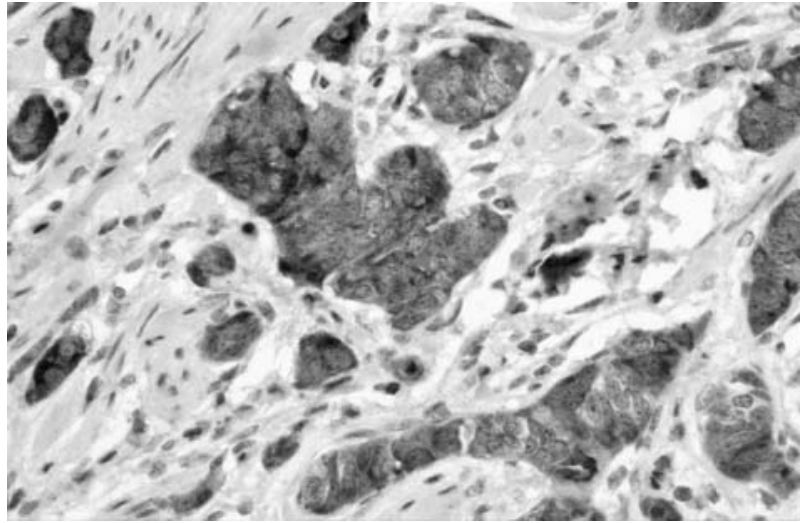

Figure 2 Gastrin releasing peptide receptor expression in a carcinoid of the appendix. Staining is diffuse and cytoplasmic (original magnification, $\times 100$ ).

it has been shown to be mitogenic in a variety of nontransformed and neoplastic, tumour derived cell lines. ${ }^{21-24}$ In vitro studies show that this growth stimulatory effect is mediated through the high affinity GRPR, which activates several intracellular signalling pathways after ligand binding. ${ }^{31}$ These include activation of phospholipase C, release of intracellular calcium, activation of protein kinase $\mathrm{C}$, and tyrosine phosphorylation.

The normal tissue distribution of GRP is restricted to the central nervous system, neuroendocrine cells of the fetal lung, and nerve fibres in the myenteric plexus of the gastrointestinal tract. ${ }^{18}$ In our study, we confirm the presence of immunoreactive GRP in the nerve cells of the myenteric plexus in the small bowel and appendix. As in previous studies, we were unable to demonstrate expression in the normal endocrine cell population of the gut. The normal distribution of the GRPR is more controversial. Although some workers report GRPRs throughout the gastrointestinal tract, including the myenteric plexus, smooth muscle coat, and normal colonic epithelium, other groups have consistently failed to show receptor expression outside the gastric antrum. ${ }^{32}{ }^{33}$ Some of this variability may result from the different techniques used to demonstrate the receptor, which have included immunohistochemistry, mRNA in situ hybridisation, reverse transcriptase polymerase chain reaction, and ligand binding studies. In a previous immunocytochemical study of the small bowel, appendix, and colon, we were able to demonstrate GRPR expression in Paneth cells but not neuroendocrine or surface epithelial cells (E J Cartwright, et al, unpublished data, 2003). In contrast to the absence of GRP/GRPR immunoreactivity in normal endocrine cells, we have found that nine of 19 carcinoid tumours aberrantly express GRP and 22 of 26 expressed the GRPR. In 1984, Bostwick et al described a series of 20 intestinal carcinoid tumours that were immunopositive for GRP. ${ }^{17}$ They found that $60 \%$ of tumours were GRP positive, although immunoreactivity was less common in colonic neoplasms (20\%) than in tumours of the appendix and ileum (73\%). In a subsequent paper, they described the staining as cytoplasmic, with intensity varying from cell to cell. ${ }^{35}$ Our findings are similar, with frequent, strong expression of GRP in small bowel carcinoids, but no staining in two colonic tumours. Unlike Bostwick et al, we also found only a low level of expression in tumours of the appendix. The distribution of staining within positive tumours was often heterogeneous, with single cells or small groups of cells being strongly positive but surrounded by unstained tumour. There was no morphological difference between the positive and negative tumour cells. In particular, there was no evidence of increased mitotic activity in these immunopositive foci. In future studies, however, it would be interesting to double label these cells with a proliferation marker, such as Ki67 or proliferating cell nuclear antigen.

"Our results suggest that although increased gastrin releasing peptide (GRP) synthesis may be involved in autocrine/paracrine stimulation of some carcinoids, overexpression of GRP receptor on its own may be sufficient to drive proliferation in others"

As far as we are aware, there have been no previous immunocytochemical studies of GRPR expression in human carcinoid tumours, although the aberrant expression of GRPR has been demonstrated in gastric and colonic adenocarcinomas. ${ }^{27}{ }^{36}$ In our current study, 22 of 26 carcinoid tumours expressed GRPR. Immunoreactivity was usually more diffuse within the tumour than the corresponding GRP staining. An interesting and perverse finding was that expression tended to be stronger in appendix and colonic tumours than in small bowel carcinoids, the reverse of the pattern seen for GRP. The correlation coefficient between GRP and GRPR immunoscores was negative ( $p=0.207$; not significant) Only one third of tumours strongly expressed both proteins. This suggests that although increased GRP synthesis may be involved in autocrine/paracrine stimulation of some carcinoids, overexpression of GRPR on its own may be sufficient to drive proliferation in others. This would be analogous to the role of c-neu (c-erb B2) in transgenic mice, where abnormal expression of a growth factor receptor is sufficient to cause mammary carcinogenesis. ${ }^{37}$ Alternatively, another ligand may activate GRPR in GRP negative neoplasms.

The staining for GRPR was uniformly granular and cytoplasmic, identical to that described in an immunohistochemical study of colon carcinomas ${ }^{27}$ Because this protein is a cell surface receptor that normally spans the plasma membrane, one would expect a membranous pattern of immunostaining. It may be that the receptor detected by this antibody is abnormally distributed within the cell as a result of either overproduction or mutation. The last option is certainly possible, because Carroll et al recently demonstrated

Table 1 GRP and GRPR expression in carcinoid tumours

\begin{tabular}{|c|c|c|c|c|}
\hline \multirow[b]{2}{*}{ Site } & \multicolumn{2}{|l|}{ GRP } & \multicolumn{2}{|l|}{ GRPR } \\
\hline & Score $\geqslant 6$ & Mean score (SD) & Score $\geqslant 6$ & Mean score (SD) \\
\hline Stomach & $0 / 1$ & 1 & $1 / 1$ & 6 \\
\hline Small intestine & $7 / 9$ & $5.53(1.23)$ & $9 / 12$ & $5.79(1.44)$ \\
\hline Appendix & $2 / 7$ & $2.86(3.00)$ & $9 / 10$ & $11(1.85)$ \\
\hline Colon & $0 / 2$ & 1 & $3 / 3$ & 7.67 \\
\hline Total & $9 / 19(47 \%)$ & $4.29(3.23)$ & $22 / 26(85 \%)$ & $7.38(3.96)$ \\
\hline
\end{tabular}

GRP, gastrin releasing peptide; GRPR, gastrin releasing peptide receptor. 


\section{Take home messages}

- Gastrin releasing peptide (GRP) was detected in nine of 19 carcinoid tumours and the GRP receptor (GRPR) in 22 of 26

- Coexpression of both the ligand and receptor was seen in seven of 19 cases

- GRPR but not GRP was more strongly expressed in appendix and colonic tumours

- Thus, an autocrine/paracrine pathway may exist for GRP stimulated cell proliferation in some of these neoplasms, analogous to that seen in small cell anaplastic carcinoma of the lung

multiple mutations of the receptor in $75 \%$ of gastric carcinomas expressing the protein..$^{38}$ They found that whereas some of these mutations caused constitutive activation of the receptor, others rendered it non-functional. Unfortunately, the intracellular location of the mutated receptor was not described. Some growth factor receptors become internalised after ligand binding and activation (for example, vascular endothelial growth factor receptor 2 (KDR)). ${ }^{39}$ Therefore, cytoplasmic staining may also reflect translocation to the cell interior as a result either of constitutive activation (an activating mutation) or binding of GRP.

In conclusion, our study has confirmed the synthesis of the GRP peptide in almost half of the gastrointestinal carcinoid tumours studied, and shown for the first time that some of these tumours coexpress the GRPR. Although it was not confirmed that the receptor was functional in these cells, our results suggest that autocrine/paracrine stimulation of tumour cell division could exist in intestinal carcinoid tumours, similar to that seen in small cell anaplastic carcinoma.

\section{ACKNOWLEDGEMENTS}

We are very grateful to A Lee for her help in the preparation of this manuscript.

\section{Authors' affiliations}

N Scott, E Millward, Department of Pathology, St James's University Hospital, United Leeds Teaching Hospitals NHS Trust, Leeds LS9 7TF, UK E J Cartwright, P L Coletta, Department of Molecular Medicine, St James's University Hospital

S R Preston, Department of Surgery, St James's University Hospital

\section{REFERENCES}

1 Wick MR. Neuroendocrine neoplasia. Am J Clin Pathol 2000;113:331-5.

2 Modlin IM, Sandor A. An analysis of 8305 cases of carcinoid tumors. Cancer 1997;79:813-29.

3 Kloppel G, Heitz PU, Capella C, et al. Pathology and nomenclature of human gastrointestinal neuroendocrine (carcinoid) tumors and related lesions. World J Surg 1996;20:132-41.

4 Anderson JR, Wilson BG. Carcinoid tumours of the appendix. $\mathrm{Br} J$ Surg 1985;72:545-6.

5 Burke AP, Thomas RM, Elsayed AM, et al. Carcinoids of the jejunum and ileum. Cancer 1997;79:1086-93.

6 Rindi G, Bordi C, Rappel S, et al. Gastric carcinoids and neuroendocrine carcinomas: pathogenesis, pathology and behaviour. World J Surg 1996;20:168-72.

7 Goddard MJ, Lonsdale RN. The histogenesis of appendiceal carcinoid tumours. Histopathology 1992;20:345-9.

8 D'Adda T, Keller G, Bordi C, et al. Loss of heterozygosity in 11q 13-14 regions in gastric neuroendocrine tumors not associated with multiple endocrine neoplasia type 1 syndrome. Lab Invest 1999;79:671-7.
9 Kytola S, Hoog A, Nord B, et al. Comparative genomic hybridization identifies loss of 18q22 qter as an early and specific event in tumorigenesis of midgut carcinoids. Am J Pathol 2001;158:1803-8.

10 Pizzie S, D'Adda T, Azzoni C, et al. Malignancy associated allelic losses on the $\mathrm{X}$ chromosome in foregut but not in midgut endocrine tumours. J Pathol 2002; 196:401-7.

11 Azzoni C, Doglioni C, Viale G, et al. Involvement of BCL-2 oncoprotein in the development of enterochromaffin-like cell gastric carcinoids. Am J Surg Pathol 1996;20:433-41.

12 Kawahara M, Kammori M, Kanauchi H, et al. Immunohistochemical prognostic indicators of gastrointestinal carcinoid tumours. Eur J Surg Oncol 2002;28:140-6.

13 O'Dowd G, Gosney JR. Absence of overexpression of p53 protein by intestinal carcinoid tumours. J Pathol 1995;175:403-4.

14 Cheng J, Sheu L, Meng C, et al. Expression of p53 protein in colorectal carcinoids. Arch Surg 1996;131:67-70.

15 Ramnani DM, Wistuba II, Behrens C, et al. K-ras and p53 mutations in the pathogenesis of classical and goblet cell carcinoids of the appendix. Cancer 1999;86:14-21.

16 Krishnamurthy S, Dayal Y. Immunohistochemical expression of transforming growth factor alpha and epidermal growth factor receptor in gastrointestinal carcinoids. Am J Surg Pathol 1997;21:327-33.

17 Bostwick DG, Roth KA, Barchas JD, et al. Gastrin-releasing peptide immunoreactivity in intestinal carcinoids. Am J Clin Pathol 1984;82:428-31

18 Spindel ER, Giladi E, Segerson TP, et al. Bombesin-like peptides: of ligands and receptors. Recent Prog Horm Res 1993;48:365-91.

19 Upp JR, Maclellan DG, Poston GJ, et al. Mechanisms of trophic action of bombesin on the pancreas. Dig Dis Sci 1986;31:1152.

20 Chu KU, Evers BM, Ishizuka J, et al. Role of bombesin on gut mucosal growth. Ann Surg 1995;222:94-100.

21 Moustafa A, Tsao M, Battey JF, et al. Expression of the gastrin-releasing peptide receptor confers a growth response to bombesin in immortalized human bronchial epithelial cells. Cancer Res 1995;55:1853-5.

22 Nelson J, Donnelly M, Walker B, et al. Bombesin stimulates proliferation of human breast cancer cells in culture. $\mathrm{Br} J$ Cancer 1991;63:933-6.

23 Bold RJ, Lowry PS, Ishizuka J, et al. Bombesin stimulates the in vitro growth of a human gastric cancer cell line. J Cell Physiol 1994;161:519-25.

24 Radulovic S, Miller G, Schally AV. Inhibition of growth of HT-29 human colon cancer xenografts in nude mice by treatment with bombesin/gastrin releasing peptide antagonist. Cancer Res 1991;51:6006-9.

$25 \mathrm{Kim} \mathrm{S}, \mathrm{Hu}$ W, Kelly DR, et al. Gastrin-releasing peptide is a growth factor for human neuroblastoma. Ann Surg 2002;235:621-30.

26 Cuttitta F, Carney DN, Mulshine J, et al. Bombesin-like peptides can function as autocrine growth factors in human small-cell lung cancer. Nature 1985;316:823-6.

27 Carroll RE, Matkowskyk KA, Chakrabarti S, et al. Aberrant expression of gastrin releasing peptide and its receptor by well differentiated colon cancers in humans. Am J Physiol 1999;276:6655-5.

28 Karnes WE, Shattuck-Brandt R, Burgart $\amalg$, et al. Reduced COX-2 protein in colorectal cancer with defective mismatch repair. Cancer Res 1998;58:5473-7.

29 Sunday ME, Kaplan LM, Motoyama E, et al. Gastrin-releasing peptide (mammalian bombesin) gene expression in health and disease. Lab Invest 1988;59:5-24.

30 Xiao D, Wang J, Hampton LL, et al. The human gastrin-releasing peptide receptor gene structure, its tissue expression and promoter. Gene $2001 ; 264: 95-103$

31 Charlesworth A, Broad S, Rozengurt E. The bombesin/GRP receptor transfected into rat-1 fibroblasts couples to phospholipase $C$ activation. Oncogene 1996;12:1337-45.

32 Chave HS, Gough AC, Palmer K, et al. Bombesin family receptor and ligand gene expression in human colorectal cancer and normal mucosa. Br J Cancer 2000;82:124-30.

33 Ferris HA, Carroll RE, Lorimer DL, et al. Location and characterization of the human GRP receptor expressed by gastrointestinal epithelial cells. Peptides 1997; 18:663-72.

34 Withdrawn.

35 Bostwick DG, Bensch KG. Gastrin-releasing peptide in human neuroendocrine tumours. J Pathol 1985; 147:237-44.

36 Preston SR, Woodhouse LF, Jones-Blackett S, et al. High affinity binding sites for gastrin-releasing peptide on human gastric cancer and Menetrier's mucosa. Cancer Res 1993;53:5090-2.

37 Muller WJ, Sinn E, Pattengale PK, et al. Single step induction of mammary adenocarcinoma in transgenic mice bearing the activated c-neu oncogene. Cell 1988;54:105

38 Carroll RE, Carroll R, Benya RV. Characterization of gastrin-releasing peptide receptors aberrantly expressed by non-antral gastric adenocarcinomas. Peptides 1999;20:229-37.

39 Stewart $M$, Turley $\mathrm{H}$, Cook N, et al. The angiogenic receptor KDR is widely distributed in human tissues and tumours and relocates intracellularly on phosphorylation. An immunohistochemical study. Histopathology 2003;43:33 